BULL. AUSTRAL. MATH. SOC.

VOL. $19(1978), 51-58$.

\title{
An existence theorem \\ for the generalized \\ complementarity problem
}

\section{J. Parida and B. Sahoo}

Given a closed, convex cone $S$, in $R^{n}$, its polar $S^{*}$ and a mapping $g$ from $R^{n}$ into itself, the generalized nonlinear complementarity problem is to find a $z \in R^{n}$ such that

$$
\begin{gathered}
g(z) \in S^{*}, z \in S, \\
\langle g(z), z\rangle=0 .
\end{gathered}
$$

Many existence theorems for the problem have been established under varying conditions on $g$. We introduce new mappings, denoted by $J(S)$-functions, each of which is used to guarantee the existence of a solution to the generalized problem under certain coercivity conditions on itself. A mapping $g: S \rightarrow R^{n}$ is a $J(S)$-function if

$$
\begin{gathered}
g(z)-g(0) \in S^{*}, \quad z \in S, \\
\langle g(z)-g(0), z\rangle=0,
\end{gathered}
$$

imply that $z=0$. It is observed that the new class of functions is a broader class than the previously studied ones.

\section{Introduction}

The generalized nonlinear complementarity problem is to find a $z \in R^{n}$ satisfying

Received 23 May 1978. 


$$
\begin{gathered}
g(z) \in S^{*}, z \in S, \\
\langle g(z), z\rangle=0,
\end{gathered}
$$

where $S$ is a closed, convex cone in $R^{n}, S^{*}$ the polar cone, and $g$ is a mapping from $R^{n}$ into itself. Problem (1.1) has been studied by Habetler and Price [2], Karamardian [3, 5], and Moré [6], who have given many theorems for the existence and uniqueness of a solution to the problem.

In this paper we introduce a nonlinear generalization of $J(S)$ matrices (which are known as $J$-matrices in [7]), denoted by $J(S)$ functions, and study problem (1.1) defined by members of this class of functions. We show that the classes of $P_{-}$and regular functions become proper subclasses of this class when $S$ is taken as $R_{+}^{n}$. We also show that a function which is strongly $S$-copositive on $S$ is a $J(S)$-function.

We establish the following: let $S$ be pointed, and let $g: S+R^{n}$ be a $J(S)$-function. Then there is a solution to (1.I) if

(i) the map $G(z)=g(z)-g(0)$ is continuous and positively homogeneous of some degree on $S$, and

(ii) the system

$$
0 \neq z \in S, G(z)+p \in S^{*},\langle G(z)+p, z\rangle=0
$$

is inconsistent for some $p \in$ int $S^{*}$.

\section{Notations and definitions}

For brevity, we shall use much of the notation of [7].

A nonempty subset $S$ of $R^{n}$ is a closed, convex cone if $S$ is closed, and $\alpha x+\beta y$ belongs to $S$ for all $\alpha, \beta \geq 0$, and $x, y \in S$. The polar cone of $S$ is the cone $S^{*}$ defined by

$$
S^{*}=\left\{x \in R^{n}:\langle x, y\rangle \geq 0 \text { for all } y \in S\right\} \text {. }
$$

A cone is said to be pointed if whenever $x \neq 0$ is in the cone, $x$ is not in the cone. For a closed, convex cone $S$, the interior of $S^{*}$, denoted by int $S^{*}$, is nonempty if, and only if, $S$ is pointed. The trivial cone $S=\{0\}$ is excluded from the discussion. 
A map $g: R_{+}^{n} \rightarrow R^{n}$ is a uniform $P$-function if there exists a scalar $c>0$ such that, for any $x \neq y$ in $R_{+}^{n}$, there is an index $k=k(x, y)$ with

$$
\left(x_{k}-y_{k}\right)\left(g_{k}(x)-g_{k}(y)\right) \geq c\|x-y\|^{2} \text {. }
$$

A map $G: R_{+}^{n} \rightarrow R^{n}$ with $G(0)=0$ is a regular function if the system

$$
\begin{gathered}
G_{i}(x)+t=0 \text { for } i \in I_{+}(x), \\
G_{i}(x)+t \geq 0 \text { for } i \in I_{0}(x), \\
0 \neq x \geq 0, t \geq 0,
\end{gathered}
$$

is inconsistent. Here $I_{+}(x)$ and $I_{0}(x)$ denote the set of indices corresponding to the positive and zero components of $x$, respectively.

A map $g: S \rightarrow R^{n}$ is strongly $S$-copositive on $S$ if there exists a scalar $c>0$ such that, for all $x \in S$,

$$
(g(x)-g(0), x) \geq c\|x\|^{2} .
$$

A map $g: S \rightarrow R^{n}$ is a $J(S)$-function if

$$
\begin{gathered}
g(x)-g(0) \in S^{*}, x \in S, \\
\langle g(x)-g(0), x\rangle=0,
\end{gathered}
$$

imply that $x=0$.

A map $G: S \rightarrow R^{n}$ is positively homogeneous of degree $d$ over $S$ if, for every $x \in S$,

$$
G(\lambda x)=\lambda^{d} G(x) \text { for all } \lambda \geq 0 \text {. }
$$

A square matrix $A$ is a $J(S)$-matrix (termed as $J$-matrix in [7]) if

$$
A x \in S^{*}, x^{T} A x=0, x \in S,
$$

imply that $x=0$. 


\section{Main results}

LEMMA 3.1. Let $S$ be a closed, convex cone in $R^{n}$, and let $g: S \rightarrow R^{n}$.

(a) If $g$ is strongly S-copositive on $S$, then $g$ is a $J(S)$ function.

(b) $g$ is a $J\left(R_{+}^{n}\right)$-function whenever either

(i) $g$ is a uniform P-function on $R_{+}^{n}$, or

(ii) the map $G(x)=g(x)-g(0)$ is a regutar function.

Proof. (a) Let $g$ be strongly $S$-copositive on $S$. Then $x \in S$, $g(x)-g(0) \in S^{*},\langle g(x)-g(0), x\rangle=0$ imply that

$$
0=\langle g(x)-g(0), x\rangle \geq c\|x\|^{2},
$$

and consequently, $x=0$.

(b) If $g$ is a uniform $P$-function on $R_{+}^{n}$, then, for every $0 \neq x \geq 0$, we have an index $k$ (depending upon $x$ ) with

$$
x_{k}\left(g_{k}(x)-g_{k}(0)\right) \geq c\|x\|^{2}>0 \text {. }
$$

The conclusion (b) for uniform $P$-function is then obvious.

To prove the second part of $(b)$ we proceed as follows: let $G(x)=g(x)-g(0)$. The consistency of the system

$$
x \geq 0, g(x)-g(0) \geq 0,\langle g(x)-g(0), x\rangle=0,
$$

implies that $x_{i}\left(g_{i}(x)-g_{i}(0)\right)=0$ for all $1 \leq i \leq n$. If $x \neq 0$, we will have $G_{i}(x)=0$ for $i \in I_{+}(x)$ and $G_{i}(x) \geq 0$ for $i \in I_{0}(x)$. Now taking $t=0$ in the definition of regular function, we get a contradiction to the regularity of $G(x)$.

REMARK 3.2. It is interesting to examine the following two examples in order to see that the classes of uniform $P_{-}$and regular functions are proper subclasses of the class of $J(S)$-functions when $S=R_{+}^{n}$. The mapping $g(x)=\left[x_{1}+x_{2}^{2}, x_{2}\right]^{T}$ defined over $R_{+}^{2}$ is a $J\left(R_{+}^{2}\right)$-function, but 
it is not a uniform $P$-function, since if $x^{k}=\left[1-2 / k, k+1 / k^{2}\right]^{T}$ and $y^{k}=[1, k]^{T}, k=2,3, \ldots$, then

$$
\begin{aligned}
& \left\{x_{1}^{k}-y_{1}^{k}\right\}\left\{g_{1}\left(x^{k}\right)-g_{1}\left(y^{k}\right)\right\}=-2 / k^{5}<0, \\
& \left(x_{2}^{k}-y_{2}^{k}\right\}\left\{g_{2}\left(x^{k}\right)-g_{2}\left(y^{k}\right)\right\}=1 /\left(4 k^{2}+1\right) \cdot\left\|x^{k}-y^{k}\right\|^{2} .
\end{aligned}
$$

The other example is the mapping $g(x)=\left[x_{2}^{2}-x_{1}, x_{2}\right]^{T}$ which is a $J\left(R_{+}^{2}\right)-$ function, but not regular.

We shall need the following results.

LEMMA 3.3 [2, Lemma 5.1]. Let $S$ be a pointed, closed, convex cone in $R^{n}$, and let $p \in$ int $S^{*}$. Then the set

$$
V=\{x: x \in S,\langle p, x\rangle=1\}
$$

is compact.

THEOREM 3.4 [7, Theorem 3.6]. If $F: R^{n} \rightarrow R^{n}$ is a continuous mapping on the nonempty, compact, convex set $C$ in $R^{n}$, then there is an $x^{0}$ such that

$$
\left\langle F\left(x^{0}\right), x-x^{0}\right\rangle \geq 0 \text { for alz } x \in C .
$$

Now we give the following existence theorem.

THEOREM 3.5. Let $S$ be a pointed, closed, convex cone in $R^{n}$, and let $g: S \rightarrow R^{n}$ be a $J(S)$-function. Then there is a solution to (1.1) if

(i) the map $G(z)=g(z)-g(0)$ is continuous and positively homogeneous of degree $d>0$ on $S$, and

(ii) there exists a vector $p \in$ int $S^{*}$ such that the system (A) $0 \neq z \in S, G(z)+p \in S^{*},(G(z)+p, z)=0$ is inconsistent.

Proof. It is easy to show that the product set $K=S \times R_{+}$is a pointed, closed, convex cone in $R^{n+I}$, and its polar is $K^{*}=S^{*} \times R_{+}$. It is also obvious that $(p, 1) \in$ int $K^{*}$. Let 


$$
C=\{(z, t): z \in S, t \geq 0,\{p, z)+t=1\} .
$$

The set $C$ is a nonempty, convex subset of $K$, and by Lemma 3.3, it is compact. Define the map $F: K \rightarrow R^{n+1}$ by

$$
F(z, t)=\left[\begin{array}{c}
G(z)+t(p+g(0)) \\
t
\end{array}\right] \text {. }
$$

It follows from Theorem 3.4 that there exists $(\bar{z}, \bar{t})$ in $C$ such that, for all $(z, t) \in C$,

$$
\langle G(\bar{z})+\bar{t}(p+g(0)), z-\bar{z}\rangle+\bar{t}(t-\bar{t}) \geq 0 .
$$

But this means that

$$
\langle G(\bar{z})+\bar{t}(p+g(0)), \bar{z}\rangle+\bar{t}^{2}=\min _{(z, t) \in C}\langle G(\bar{z})+\bar{t}(p+g(0)), z\rangle+\bar{t} \cdot t .
$$

Now using the Kuhn-Tucker necessary conditions of optimality [1] for cone domains, we have a $\zeta_{0}$ in $R$ such that

$$
\begin{gathered}
G(\bar{z})+\bar{t}(p+g(0))+\zeta_{0} p \in S^{*}, \bar{t}+\zeta_{0} \geq 0, \\
\left\langle G(\bar{z})+\bar{t}(p+g(0))+\zeta_{0} p, \bar{z}\right\rangle=0, \bar{t}\left(\bar{t}+\zeta_{0}\right)=0 \\
\bar{z} \in S, \bar{t} \geq 0,\langle p, \bar{z}\rangle+\bar{t}=1 .
\end{gathered}
$$

We claim that $\bar{t}>0$. If not so, then suppose that $\bar{t}=0$. This with (1.2) implies that $\bar{z} \neq 0$, and consequently, we have the system

$$
\begin{gathered}
G(\bar{z})+\zeta_{0} p \in S^{*}, \quad \zeta_{0} \geq 0, \\
\left\langle G(\bar{z})+\zeta_{0} p, \bar{z}\right\rangle=0,
\end{gathered}
$$

consistent for $0 \neq \bar{z} \in S$. Since $g$ is a $J(S)$-function, $\zeta_{0}$ can not be equal to zero. Also, $\zeta_{0} \ngtr 0$ since, in that case, taking the positively homogeneous property of $G$ into consideration we can have a vector $0 \neq \bar{y}=\bar{z} /\left(\zeta_{0}\right)^{1 / d} \in S$ satisfying the system $(A)$. Hence, $\bar{t}>0$, and consequently, we have $\bar{t}+\zeta_{0}=0$. Now substituting $\zeta_{0}=-\bar{t}$ in (1.2), it can be easily shown that $z^{0}=\bar{z} /(\bar{t})^{1 / d}$ is the desired solution.

REMARK 3.6. If we take $S=R_{+}^{n}$ and $p=e \alpha, \alpha>0$, with 
$e^{T}=(1,1, \ldots, 1)$, and assume that $G(z)=g(z)-g(0)$ is regular, then Theorem 3.5 reduces to Theorem 3.1 in [4]. If we take $g(z)=M(z)+q$ where $M: S \rightarrow R^{n}$ is a nonlinear map with $M(0)=0$, and $q$ is a vector in $R^{n}$, then Theorem 3.5 yields Theorem 3.1 in [5]. Theorem 4.2 of Parida and Sahoo [7] follows as a special case of Theorem 3.5 .

REMARK 3.7. We consider it interesting to provide the following example. Let $g: R^{3} \rightarrow R^{3}$ be an affine map defined by $g(x)=\left[x_{1}-x_{2}, x_{1}+x_{2}, x_{3}-1\right]^{T}$. The problem is to find a solution to the system

$$
\begin{gathered}
y=g(x), x_{1}^{2}+x_{2}^{2}-d^{2} x_{3}^{2} \leq 0, x_{3} \geq 0, \\
y_{1}^{2}+y_{2}^{2}-y_{3}^{2} / d^{2} \leq 0, y_{3} \geq 0, d \neq 0, \\
x^{T} y=0 .
\end{gathered}
$$

It can be cast in the form (1.1) as follows: find $x \in R^{n}$ such that

$$
x \in S, g(x) \in S^{*},\langle g(x), x\rangle=0,
$$

where

$$
\begin{aligned}
S & =\left\{x \in R^{3}: x^{T} B x \leq 0, x_{3} \geq 0\right\}, \\
S^{*} & =\left\{y \in R^{3}: y^{T} B^{-1} y \leq 0, y_{3} \geq 0\right\},
\end{aligned}
$$

and

$$
B=\left[\begin{array}{ccc}
1 & 0 & 0 \\
0 & 1 & 0 \\
0 & 0 & -d^{2}
\end{array}\right], \quad d \neq 0 .
$$

It can be shown that $g$, as given above, with $p=[0,0,1]^{T}$ satisfies the conditions of Theorem 3.5. So there exists a solution to the problem. Indeed, we find that $x_{1}=0, x_{2}=0, x_{3}=1$ is a solution. 


\section{References}

[1] M.S. Bazaraa and J.J. Goode, "Necessary optimality criteria in mathematical programming in the presence of differentiability", J. Math. Anal. Appl. 40 (1972), 609-621.

[2] G.J. Habetler and A.L. Price, "Existence theory for generalized nonlinear complementarity problems", J. Optimization Theory Appl. 7 (1971), 223-239.

[3] S. Karamardian, "Generalized complementarity problem", J. Optimization Theory App 2. 8 (1971), 161-168.

[4] S. Karamardian, "The complementarity problem", Math. Progranoming 2 (1972), 107-129.

[5] S. Karamardian, "An existence theorem for the complementarity problem", J. Optimization Theory Appl. 19 (1976), 227-232.

[6] Jorge J. Moré, "Coercivity conditions in nonlinear complementarity problems", SIAM Rev. 16 (1974), I-I6.

[7] J. Parida and B. Sahoo, "A note on generalised linear complementarity problems", Bull. Austral. Math. Soc. 18 (1978), 161-168.

Department of Mathematics,

Regional Engineering College,

Rourkela,

Orissa,

India. 